Ingrid Schröder (Hamburg)

\title{
Sprachbiografie und Spracheinstellung. Niederdeutsch als Mittel der Identitätsstiftung in der Großstadt?
}

\begin{abstract}
Im Projekt „Einstellungen gegenüber regionalen Sprachformen in der Großstadt: Niederdeutsch in Hamburg“ soll geklärt werden, inwieweit regionale Sprachformen in einer Metropole als Kennzeichen stadttypischer Räume und Einrichtungen im Bewusstsein der Bewohner verankert sind. Im Mittelpunkt des Beitrags steht der theoretisch-methodische Rahmen der Studie mit einem Modell, das die Bedeutung von Spracheinstellungen und Sprachbiografie im Verhältnis zu Identitätskonstruktionen fokussiert und damit den Analyserahmen darstellt. Anhand von Beispielen wird der Zusammenhang von Spracheinstellungen, Ortseinstellungen, Biografie und darauf bezogenen Identitätskonstruktionen am stereotyp ausgeprägten Themenkomplex „Niederdeutsch als Hafensprache“/,Niederdeutsch spricht man im Hafen“ erläutert.
\end{abstract}

\section{Einleitung}

GP 58: Ja, ich sag einfach mal, Plattdeutsch hat für Hambuich schon eine Bedeutung ((.)) ähm Plattdeutsch ist auch ein Stück Hambuich. Und meines Erachtens gehört die plattdeutsche Sprache ähm die gehört einfach zu Hambuich, [I: Hmhḿ.] die gehört einfach auch zum Hafen, die gehört einfach zum Milieu I: Ja. GP 58: Hambuich [I: Hmhḿ.] gehört diese Sprache einfach dazu. (GP 58, 896-898) ${ }^{1}$

1 Die Stellenangaben beziehen sich auf die Zählung der Events in den mit EXMARaLDA erstellten Transkripten. - GP = Gewährsperson; I = Interviewer/Interviewerin. Simultan einsetzende Äußerungen oder Hörerrückmeldungen werden mit Sprecherangabe (GP, I) unmittelbar hinter dem Lexem, mit welchem die Simultaneität endet, in eckigen Klammern angegeben. Unterstreichungen kennzeichnen betonte Wörter oder Wortteile. Non- oder paraverbale Handlungen werden mit doppelten Klammern kenntlich gemacht. Treten sie parallel zu Äußerungen eines Sprechers auf, ist dies an eckigen Klammern zu erkennen. Die Angabe folgt dann auf das letzte simultan gesprochene Lexem. Durch doppelte Klammern umschlossene Punkte stehen für kürzere Sprechpausen, wobei ((.)) Pausen von bis zu einer Sekunde, ((..)) Sprechpausen von circa zwei Sekunden und ((...)) Sprechpausen von circa drei Sekunden kennzeichnen. In einfache runde Klammern gesetzte Teile sind nicht klar verständlich. 
Dieses Zitat aus dem Interviewkorpus des Projekts „Einstellungen gegenüber regionalen Sprachformen in der Großstadt: Niederdeutsch in Hamburg (NiH)“ belegt eindrücklich die Hypothese, dass Niederdeutsch in Hamburg als ein besonderes Ortsmerkmal wahrgenommen wird. Niederdeutsch wird als zugehörig zum Hafen und zu dessen Milieu, insgesamt als zugehörig zu Hamburg angesehen. Im Projekt soll der Hypothese nachgegangen werden, dass regionale Sprachformen in Metropolen als Kennzeichen stadttypischer Räume und Einrichtungen im Bewusstsein der Bewohner verankert sind und als Teil einer städtischen Identität verstanden werden, auch wenn die Sprachwirklichkeit längst eine andere geworden ist. Im Zentrum steht der sozialsymbolische Gehalt, welcher der Sprachform zugeschrieben wird, und dessen Bedeutung für die Sprecher.

Im Folgenden sollen nicht die einzelnen Ergebnisse des Projekts im Mittelpunkt stehen, sondern nach einem kurzen Projektaufriss wird der theoretischmethodische Rahmen der Studie beschrieben, wobei ein Modell zu entwickeln ist, das die Bedeutung von Spracheinstellungen und Sprachbiografie im Verhältnis zu Identitätskonstruktionen fokussiert und den Analyserahmen darstellt. ${ }^{2}$ Anhand von Beispielen kann schlaglichtartig verdeutlicht werden, auf welche Weise die genannten Komponenten aufeinander bezogen sind und mit welchen Mitteln sie sprachlich realisiert werden.

\section{Das Projekt: Ausgangssituation, Ziele, Design}

Dem Projekt ${ }^{3}$ liegt die Beobachtung zugrunde, dass das Niederdeutsche in Hamburg - trotz rückläufiger Sprecherzahlen (vgl. Adler et al. 2016, S. 15) - in der Öffentlichkeit, in der Kultur und in den Medien eine nicht zu unterschätzende Rolle spielt. ${ }^{4}$ Die Sprachwahl Niederdeutsch, die Rezeption entsprechender kultureller Angebote, die positive Bewertung der Sprache sowie das Engagement für

2 Vgl. auch die theoretischen Vorüberlegungen in Schröder/Jürgens (2017, S. 13-26), die hier in einem Modell zusammengeführt werden, mit weiterführender Literatur.

3 Projektleitung: Ingrid Schröder; wissenschaftliche Mitarbeiterinnen: Carolin Jürgens (bis August 2015), Lara Neumann (ab November 2015), in Kooperation mit Christine Fuhrmeister (August 2016 bis Dezember 2017); studentische Hilfskräfte: Franziska Fleischhauer, André Pabst, Bernadette Schlaffner, Verena Turkowski. Projektlaufzeit: 1.6.2014-31.3.2018. Eine ausführliche Projektbeschreibung bieten Schröder/Jürgens (2017).

4 Zum Enregisterment mit Niederdeutsch in Hamburg vgl. Jürgens 2016. Auch die Verpflichtungen Hamburgs zur Förderung der Sprache im Rahmen der Europäischen Charta der Regionaloder Minderheitensprachen können als Beleg für die Zuschreibung eines hohen sozialsymbolischen Signalwerts gelten (vgl. Eichinger 1999, S. 50). 
die Verwendung des Niederdeutschen können als Ausdruck einer besonderen Verbindung zur Stadt und damit als Bestandteil einer spezifischen hamburgischen Identität verstanden werden. Im Projekt soll untersucht werden, welche Funktionen dem Niederdeutschen im Alltagsleben der Großstadt zukommen, welche Sprachkonzepte sich dabei abzeichnen und welches Identifikationspotenzial damit verbunden ist, d.h., inwieweit Niederdeutsch als Mittel der Identitätsstiftung wahrgenommen wird.

Dafür sind die Spracheinstellungen, also die Inhalte und Bewertungen, die in Hamburg mit dem Niederdeutschen verbunden sind, $\mathrm{zu}$ analysieren. $\mathrm{Zu}$ fragen ist, welche sprachbezogenen Wissensbestände vorliegen und ob gängige stereotype Zuschreibungen wie „Niederdeutsch spricht man auf dem Lande“ (vgl. Schröder/Jürgens 2016) durch spezifische städtische Stereotype wie „Niederdeutsch spricht man im Hafen“ flankiert oder auch überlagert werden. In die Analyse sind auch die Bewertungen des Niederdeutschen einzubeziehen und deren möglicher Einfluss auf den Sprachgebrauch, auf die Rezeption des Kulturangebots und auf das Engagement für die Sprachförderung. Schließlich ist dem Zusammenhang von Spracheinstellungen und Ortseinstellungen nachzugehen, um eine mögliche identitätsstiftende Funktion aufzudecken. Individuelle biografische Erfahrungen der Sprachnutzer mit dem Niederdeutschgebrauch lassen zudem erkennen, welche Sprachdomänen für sie relevant sind bzw. waren und welche Veränderungen im Lauf des Lebens eingetreten sind. ${ }^{5}$

Das Projekt umfasst zwei Teilstudien. Bei der ersten Teilstudie handelt es sich um eine qualitativ angelegte Interviewstudie. Die Datengrundlage bilden 42 narrative sprachbiografische Interviews mit insgesamt 44 Personen aus fünf verschiedenen Untersuchungsfeldern der Hamburger Öffentlichkeit, nämlich Kultur, Medien, Institutionen, Freizeit und Politik. Die Probanden sind zwischen 1932 und 1982 geboren und stammen überwiegend aus Hamburg oder Norddeutschland. Sie weisen sich selbst eine gute passive Niederdeutschkompetenz zu und sind mehrheitlich aktive Sprecher. Alle Befragten setzen sich auf unterschiedliche Weise mit dem Niederdeutschen im öffentlichen Bereich auseinander.

Um weitere Kreise der Hamburger Bevölkerung unabhängig von ihren Berührungspunkten mit dem Niederdeutschen in die Untersuchung einzubeziehen, wurde eine zweite, quantitative Teilstudie konzipiert, die sich an alle Personen richtet, die in Hamburg ihren Lebensmittelpunkt haben. Auf diese Teilstudie kann im vorliegenden Artikel nicht weiter eingegangen werden.

5 Zusätzlich zu den Einstellungen gegenüber dem Niederdeutschen werden auch die Einstellungen gegenüber dem lokalen Substandard in Hamburg in die Untersuchung vergleichend einbezogen. 


\section{Modellierung: Einstellungen, Biografie, Narration, Identität}

\subsection{Einstellungen und Biografie}

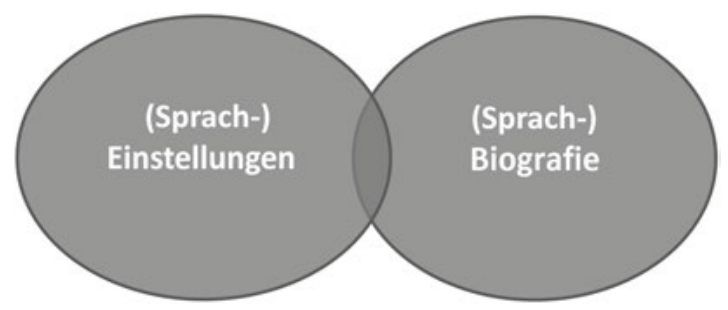

Abb. 1: Einstellungen und Biografie

Als theoretische Basis liegt der Studie ein Modell zugrunde, das Einstellungen, v.a. zu Sprache und Ort, und (Sprach-)Biografie als wesentliche Komponenten der sprachbezogenen Identitätsstiftung miteinander verknüpft. Im Folgenden soll dieser Zusammenhang erläutert werden.

\subsubsection{Einstellungen}

Einstellungen können als psychische Dispositionen beschrieben werden, als deren wesentliche Faktoren der Einstellungsträger, das Bezugselement, auf das sich die Einstellung richtet, und die Verhaltensoption gegenüber dem Bezugselement gelten (vgl. grundsätzlich Allport 1935, S. 810; Eagly/Chaiken 1998, S. 269). Die Verhaltensoption steht im engen Zusammenhang mit der Erfahrung und dem damit verbundenen (erworbenen) Wissen sowie mit der Bewertung des Bezugselements. Dies kann in einem Drei-Komponenten-Modell abgebildet werden, das kognitive (Erfahrungen und Wissen), affektive (Bewertungen) und konative Anteile (Verhaltensoptionen) umschließt (vgl. Lasagabaster 2004, S. 200; Fischer/ Wiswede 2009, S. 285). Da der jeweilige Anteil der Komponenten veränderlich ist und sich auch die Komponenten selbst im Lauf der Zeit verändern, ist dieses Modell als dynamisch aufzufassen. In der Analyse offenbaren Wissensbestände und Bewertungen die Haltungen der Einstellungsträger und erlauben es, Selbstund Fremdbilder zu ermitteln.

Angewandt auf Sprache lassen sich die kognitive Komponente auf das Wissen über sprachliche Strukturen, auf die Verwendung der Sprache und ihre Funk- 
tionen beziehen, die affektive Komponente auf die damit verbundenen Emotionen, die konative Komponente schließlich auf den Gebrauch oder die Vermeidung von Sprachformen (vgl. Neuland 1993, S. 728). Spracheinstellungen werden häufig überlagert durch Einstellungen gegenüber (Nicht-)Sprechern, d.h. gegenüber Gruppen, oder auch durch Bewertungen der Sprachsituationen (vgl. Riehl 2000, S. 141; Jürgens 2015, S. 53), die daher in der Analyse zu berücksichtigen sind. Im Projektzusammenhang spielen gleichermaßen die Ortseinstellungen eine wesentliche Rolle, nämlich das Wissen über Hamburg, die Bewertung der Stadt und die Nutzung der Stadt als Lebensraum.

Besonders deutlich kommen Einstellungen in Form von Stereotypen zum Ausdruck, die als empirisch mehr oder weniger fundierte, in der Regel übergeneralisierte Zuschreibungen aufgefasst werden können und Wissensbestände mit Bewertungen verbinden. ${ }^{6}$ Sie repräsentieren virulente gesellschaftlich geteilte Konzepte. Als verbale Ausdrücke einer bestimmten Überzeugung (Quasthoff 1987, S. 787) lassen sie sich im Einstellungsmodell der kognitiven Komponente zuordnen. Durch sie werden subjektive Bestände des Alltagswissens ausgedrückt, die jedoch nicht in jedem Fall zutreffend sein müssen (vgl. Garrett 2010, S. 31; Cuonz 2014, S. 54).

\subsubsection{Biografie}

Die Berücksichtigung der verschiedenen Lebensphasen ist grundlegend für die Analyse sprachbiografischer Daten. Jede Lebensphase ist durch ein spezifisches soziales Netzwerk geprägt, das zugleich für den Ausbau des sprachlichen Repertoires und für die Wahl der sprachlichen Mittel relevant ist, die als Teile einer personalen Identität angesehen werden können (vgl. Wirrer 2017). Biografische Daten sind insbesondere im Zusammenhang mit dem Spracherwerb, mit dem sich wandelnden Sprachgebrauch und insgesamt mit den persönlichen Erfahrungen mit Sprache von Interesse. Mit den Spracherfahrungen gehen immer auch Einstellungen unter Einschluss von Bewertungen einher, die jeweils an die einzelnen biografischen Stationen angebunden sind.

Der Unterscheidung zwischen (realem) Lebenslauf und (rekonstruierter) Biografie folgend, sind Sprachbiografien als subjektive kognitive Konstrukte beschrieben worden, die eine identitätsstiftende Funktion besitzen, nämlich als Wahrnehmung und Verarbeitung der eigenen Lebensgeschichte (vgl. Bieberstedt 2017).

6 Im Projektzusammenhang vgl. dazu Schröder/Jürgens (2016); Schröder (2013, 2010); Jürgens (2015). 
Die erinnerte Geschichte ist kein objektives Abbild der Realität, sondern spiegelt die spezifische Sicht der Person, ihre Werte sowie ihre Bewertungen wider (vgl. Tophinke 2002). Im Projekt sind beide Aspekte zu berücksichtigen, zum einen die Rekonstruktion des Sprachgebrauchs in verschiedenen Lebensphasen und zum anderen die Beurteilung dieser Entwicklung auf der Folie des individuellen Relevanzsystems.

\subsubsection{Wissensformen}

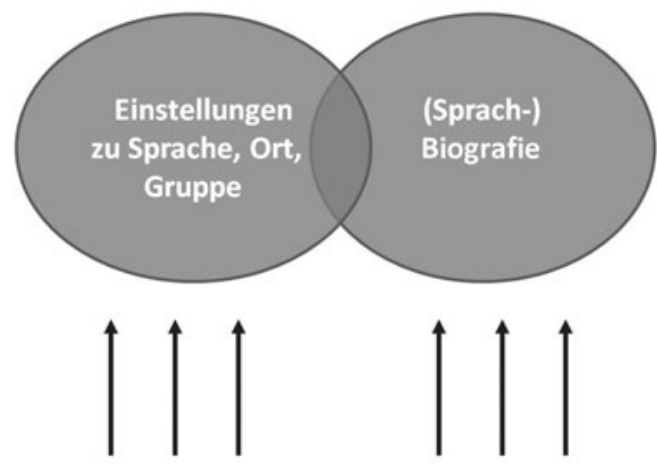

Alltagswissen:

situatives Erfahrungswissen / deklaratives Weltwissen

Abb. 2: Einstellungen, Biografie und Alltagswissen

Wie die erinnerte Biografie stellen auch die Einstellungen kognitive Konstrukte dar, die aus dem subjektiven Alltagswissen gespeist werden (vgl. Berger/Luckmann 2010, S. 140; Jürgens 2015, S. 18-60), das sich wiederum aus situativem Erfahrungswissen und deklarativem Weltwissen zusammensetzt. Situatives Erfahrungswissen besteht aus Erinnerungen an konkrete biografische Erlebnisse, deklaratives Weltwissen hingegen ist gesellschaftlich vermitteltes Wissen (Kaiser 2010, S. 1). In dieses Wissen fließen auch Bewertungen ein. 


\subsection{Narration}

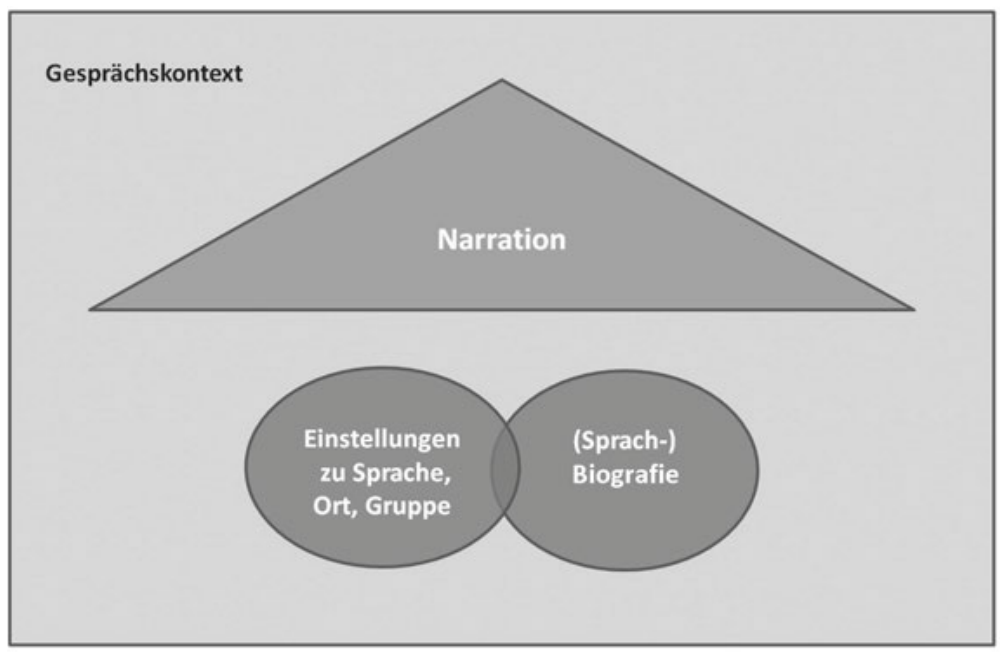

Abb. 3: Einstellungen, Biografie und Narration

Einstellungen und Biografie können als psychische Dispositionen, als Wissensund Bewertungsbestände bzw. als kognitive Konstrukte nicht ohne Weiteres wahrgenommen werden (vgl. Neuland 1993, S. 728; Jürgens 2015, S. 53). Zugänglich sind sie v.a. in Form von sprachlichen Äußerungen, die sich als Narrationen in biografischen Interviews erheben lassen.

Grundsätzlich lässt sich eine Narration im weiteren Sinne von einer Narration im engeren Sinne unterscheiden (vgl. Ehlich 1983). Zur Narration i.w.S. gehören mehrere Handlungsmuster, die im alltäglichen Sprachgebrauch dem Erzählen zugeschrieben werden, wie z.B. Berichten oder Beschreiben. Für die Narration i.e.S. ist ein spezifisches sprachliches Handlungsmuster charakteristisch. Zentral sind die Thematisierung eines ungewöhnlichen Ereignisses und die Wohlgeformtheit, die sich in der narrativen Ordnung und Verknüpfung der Ereignisse sowie dem Erreichen eines sinnstiftenden Endpunkts niederschlägt (vgl. Kraus 2002, S. 167-171, im Anschluss an Gergen/Gergen 1988). Beim sinnstiftenden Endpunkt handelt es sich aus linguistischer Perspektive um die These, die durch eine Erzählung begründet, belegt oder veranschaulicht werden soll, wie z.B. dass man Niederdeutsch im Hafen spreche. ${ }^{7}$

7 Vgl. zum Zusammenhang auch Neumann/Schröder (2017, S. 228-231), mit Beispielanalysen. 
Eine wesentliche Funktion des Erzählens liegt darin, „den Inhalt der Selbstdarstellung für Sprecher und Hörer gemeinsam zu machen, also in die gemeinsame Sprecher- und Hörerwelt zu integrieren“ (Ehlich 1983, S. 139). Dabei werden besondere sprachliche Verfahren eingesetzt, um Zustimmung $\mathrm{zu}$ erheischen, Selbstpositionierungen abzusichern und Thesen oder Bewertungen zu plausibilisieren (vgl. Lucius-Hoene/Deppermann 2002, S. 169). Somit ist eine Analyse der Narration nicht nur auf die internen Merkmale der Erzählung zu richten, sondern zugleich auf den Gesprächszusammenhang und folglich auf die Interaktion der Gesprächspartner (vgl. Tophinke/Ziegler 2006; König 2014, 2017), d.h. im Projekt auf den aktuellen Gesprächskontext des Interviews.

\subsection{Identität}

\subsubsection{Personale Identität}

Personale Identität lässt sich als das ,je spezifische Selbst- und Weltverhältnis sozialer Subjekte“, als „ihr Selbstbild und Selbstverständnis“ bestimmen (Rosa 2007, S. 47). Konstituierende Merkmale sind dabei Kontinuität, Konsistenz und Kohärenz (vgl. Straub 2011, S. 284-287). ${ }^{8}$ Kontinuität bezieht sich auf die diachrone Identität (Straub 2000, S. 172), die Einheit der Person im Wandel der Zeit und die damit verbundene Vielfalt an Erfahrungen, Konsistenz auf die „logische Verträglichkeit“ (ebd., S. 175), d.h. die Verträglichkeit verschiedener Aussagen zur Identität, und Kohärenz auf die innere Stimmigkeit der Person und damit auf den Zusammenhang der verschiedenen Rollen, in denen ein Mensch agiert (ebd.).

Da Menschen in ihren jeweiligen Rollen verschiedenen Gruppen angehören, werden auch die Werte der jeweiligen Gruppen in das eigene Identitätskonzept einbezogen. Aus Einstellungen gegenüber Gruppen wird soziale Identität gespeist (vgl. Tajfel 1981, S. 255). Als deren spezifische Form kann die raumbezogene regionale Identität angesehen werden (vgl. Mühler/Opp 2008, S. 1 f.). ${ }^{9}$ Region ist dabei als historisches, politisches, soziales und kulturelles Konzept zu verstehen, das für personale wie auch für soziale Identifikationsprozesse eine Rolle spielt

8 Vgl. auch Lucius-Hoene/Deppermann (2002, S. 48). Krappmann (2004, S. 406) unterscheidet als Komponenten Selbigkeit, Kontinuität, Einheit.

9 Unter „regionaler Identifikation“ verstehen Mühler/Opp (2008, S. 1f.) „das Ausmaß, in dem eine Person eine Region oder, genauer, die Bewohner einer Region positiv bewertet oder, anders gesagt, sich mit einer Region verbunden fühlt“. 
(vgl. Blotevogel 2001, S. 3; Flender/Pfau/Schmidt 2001, S. 24). Auch Städte können auf diese Weise identitätsstiftend sein.

Identitätskonzepte werden nicht zuletzt durch die kulturellen Spezifika bestimmt, zu denen vor allem die Sprache gehört. Sprachidentität lässt sich als Teil der personalen Identität beschreiben (vgl. Thim-Mabrey 2003, S. 2), wobei die Haltung zu einer Sprache, mithin die Spracheinstellungen, ausschlaggebend ist. ${ }^{10}$ Sie kann auch als Teil der sozialen Identität gefasst werden, wenn Sprache als Gruppenabzeichen wahrgenommen wird. Diese Symptomfunktion der Sprache kann aktiv dazu genutzt werden, Identität bzw. Teile davon hervorzuheben. ${ }^{11}$
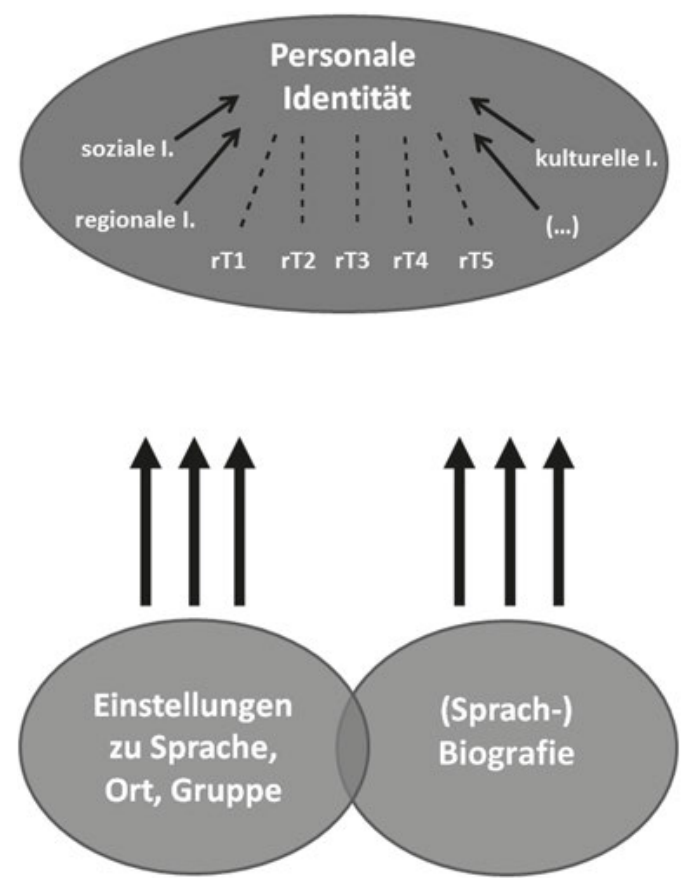

Abb. 4: Einstellungen, Biografie und personale Identität

10 Die Studien von Anne Betten zu ihrem Israelkorpus zeigen nachdrücklich die Rolle der Sprache als Ausdruck kultureller Identität (vgl. z.B. Betten 2017).

11 Zur sozialsymbolischen Funktion von Sprache vgl. Hess-Lüttich (2004, S. 492); zum Konzept der Indexikalität auch Silverstein (2003, S. 217-222). - In diesem Zusammenhang spricht Eckert (2012, S. 87) von sozialer Bedeutung, die durch die Verwendung spezifischer sprachlicher Varianten oder durch die Nutzung eines bestimmten Stils erzeugt wird. 
Da Personen in verschiedenen biografischen Rollen agieren, stehen nicht in jeder Situation alle Facetten der Identität gleichermaßen im Vordergrund. Diese Rollen zeigen sich in Form von spezifischen Teilidentitäten (rT = rollenbezogene Teilidentitäten), die mit Bezug auf die jeweilige Sprachverwendung von Kresić (2006, S. 15 ff.) als Sprach-Teil-Identitäten angesehen werden.

\subsubsection{Narrativ gestiftete Identität und Positionierung}
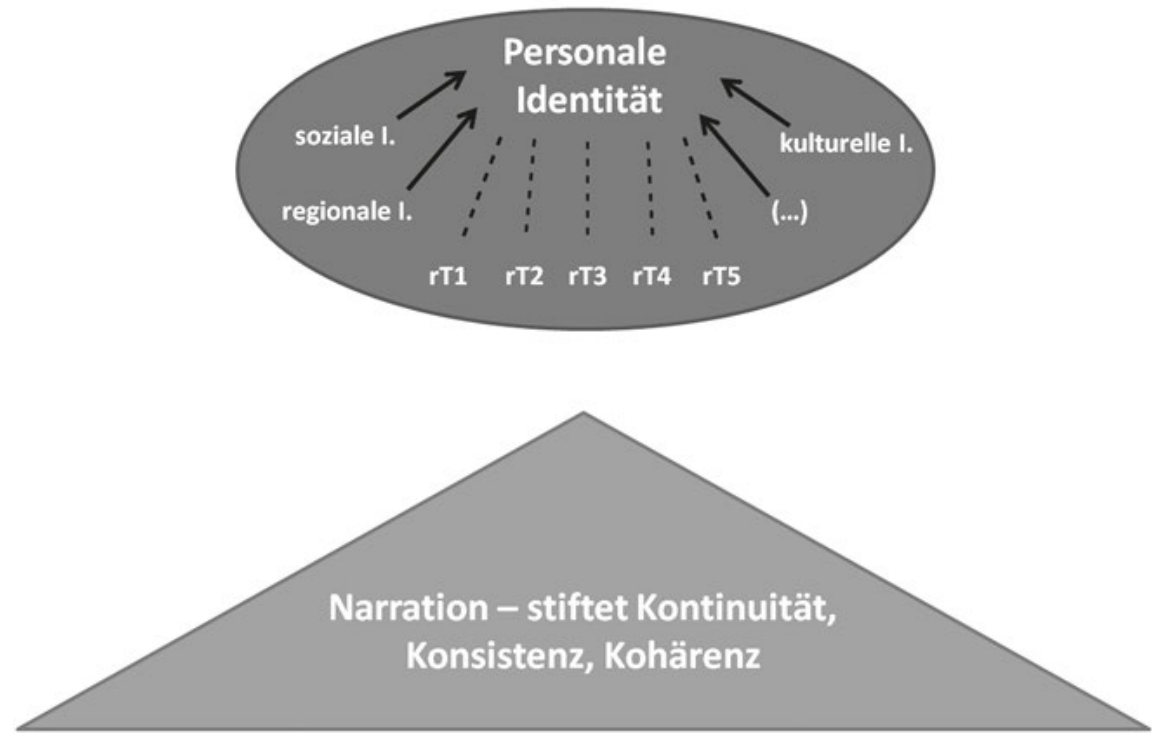

Abb. 5: Narrativ gestiftete Identität

Ebenso wenig wie Einstellungen und Biografie ist Identität der Analyse an sich zugänglich, sondern tritt ausschließlich in Form von Verhaltensweisen zutage, und zwar durch semiotische Verfahren sowohl sprachlicher wie nicht-sprachlicher Art. Insbesondere durch biografische Erzählungen können Selbstbilder gestaltet werden, indem Kontinuität, Konsistenz und Kohärenz erzählerisch hergestellt werden (vgl. Straub 2011, S. 286). Somit werden Erzählungen zu Konstruktionen der personalen Identität (vgl. Hügli 2010, S. 141). Dieser Zusammenhang wird häufig als narrative Identität gefasst, doch erscheint es angemessener, von narrativ konstruierter Identität zu sprechen. Für eine solche narrative Identitätskonstruktion sind spezifische sprachliche Mittel erforderlich, wesentlich die Bezugnahmen auf identitätsstiftende Elemente wie Sprache, Ort oder Gruppe und 
die damit verbundenen Zuschreibungen, die explizit als Propositionen oder implizit in verschiedenen sprachlichen Handlungsmustern formuliert werden können (vgl. dazu unten, Abschn. 4).

Als wesentliche Elemente solcher Identitätskonstruktionen beschreiben Lucius-Hoene/Deppermann Positionierungsverfahren als „die diskursiven Praktiken, mit denen Menschen sich selbst und andere in sprachlichen Interaktionen aufeinander bezogen als Personen her- und darstellen“ (Lucius-Hoene/ Deppermann 2004, S. 168). Dazu gehören die Attribute, Rollen, Eigenschaften und Motive, die sie im Gespräch für die Identitätskonstruktion in Anspruch nehmen. Mithilfe von Positionierungshandlungen stellen die Sprecher ihre eigene Rolle dar, sowohl im Verhältnis zu anderen Personen innerhalb der Erzählung als auch im Verhältnis zu den Kommunikationspartnern in der aktuellen Gesprächssituation. Die Identitätsarbeit ist also immer auf zwei Bereiche hin ausgerichtet, auf den Erzählinhalt und auf die Erzählsituation, d.h. den aktuellen Gesprächskontext.

\subsection{Zusammenschau: Einstellungen, Biografie, Narration und Identität}

Die Formen der Narration im weiteren Sinne und die darin eingebetteten Positionierungen gehören zu den zentralen Analysegegenständen des Projekts. Durch die Narration werden Einstellungen und Biografie versprachlicht und dadurch erst zugänglich gemacht. Zugleich wird die Identität narrativ zum Ausdruck gebracht. Das Modell stellt somit in erster Linie eine Momentaufnahme der aktuellen Gesprächssituation dar, in der bestimmte Teilidentitäten bzw. Rollen hervorgehoben werden können, die in anderen Erzählkontexten zurücktreten. Es handelt sich um einen während des Interviews durch die Narration aktuell und situationsbezogen verfertigten Identitätsentwurf.

Das Projekt stützt sich generell auf das dreigliedrige Einstellungskonzept, indem für die qualitative Studie die konative Komponente als analytischer Ausgangspunkt gewählt wurde und die sprachliche Verhaltensoption als Kriterium für die Auswahl der Gewährspersonen diente. Die Daten zu Sprachwissen (kognitive Komponente) und Sprachbewertung (affektive Komponente) wurden im Rahmen sprachbiografischer Interviews erhoben. Die darauf bezogenen Analysen geben darüber Aufschluss, welche Zuschreibungen mit dem Niederdeutschen und ferner auch mit der hamburgischen Umgangssprache verbunden werden und inwiefern diese Sprachformen zur Konstruktion der eigenen Identität beitragen. 


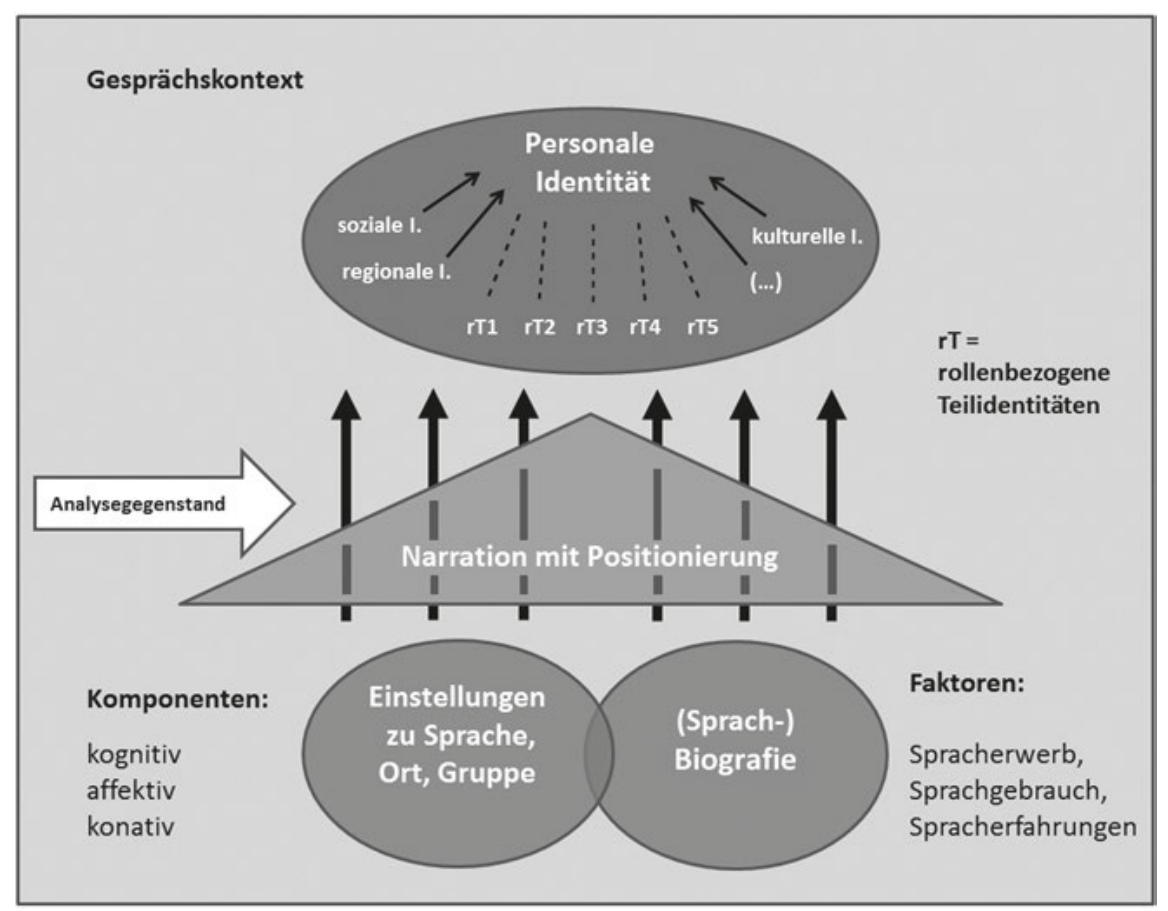

Abb. 6: Einstellungen, Biografie, Narration und Identität

Rückschlüsse auf die raumbezogene Identität erlauben die Ortseinstellungen, indem die kognitive und die affektive Komponente durch Erfragung des Ortswissens und der Ortsbewertungen fokussiert werden, während die konative Komponente wiederum als Auswahlkriterium erfasst ist (Hamburg als Wohnort bzw. Lebensmittelpunkt der Befragten). Es soll überprüft werden, ob und auf welche Art und Weise das Niederdeutsche im Sinne der Konstruktion regionaler Identität in das Stadtkonzept eingebunden wird.

Zudem sind (sprach)biografische Faktoren heranzuziehen, um die von den Probanden gewählten Verhaltensoptionen (also den Sprachgebrauch und das Engagement) erklären zu können. Auswirkungen auf die Spracheinstellungen können etwa der Erwerbsmodus als Erst- oder Zweitsprache haben, der Sprachgebrauch in der Kindheit, Erfahrungen in biografischen Umbruchsituationen oder auch einzelne individuelle Erlebnisse, die mit dem Niederdeutsch-Gebrauch verknüpft sind. 


\section{Datenanalyse}

Das Modell legt nahe, dass mehrere analytische Zugriffe methodisch miteinander zu verschränken sind. Zum einen spielen inhaltsanalytische Verfahren eine Rolle, die insbesondere bei der Annotation des Materials und der darauf folgenden inhaltlichen Strukturierung zum Tragen gekommen sind. Hier sind thematische Bereiche $^{12}$ und Lebensphasen ${ }^{13}$ zu differenzieren.

Um die biografischen Erfahrungen, die Einstellungen und die damit verbundenen Stereotype (vgl. Schröder/Jürgens 2016) sowie die Positionierungen (vgl. Neumann/Schröder 2017) zu untersuchen, wurde in Anlehnung an die Stereotypenforschung ein Schema entwickelt, das alle Sprachebenen berücksichtigt (vgl. dazu Schröder/Jürgens 2016, S. 356, mit weiterführender Literatur). Kategorisierungen, d.h. die Bezeichnungen für Sprachvarietäten, Sprechergruppen und Sprachsituationen, werden ebenso erfasst wie die damit verbundenen Zuschreibungen sowohl expliziter als auch impliziter Art. Als Ausgangspunkte der Gesprächsanalyse werden die sichtbar werdenden Wissensstrukturen (Weltwissen, Erfahrungswissen) genutzt. Vertextungsmuster auf äußerungsübergreifender Ebene spielen ebenso eine Rolle wie semantisch-pragmatische Operationen (Generalisierung, Subjektivierung u.a.), epistemische und evaluative Einstellungen der Sprecher, Formulierungsmuster (Vergleiche, Beispiele u.a.), syntaktische Konstruktionen und lexikalische Mittel. Außerdem wird die Interaktion der Gesprächspartner in den Blick genommen wie auch die Sprachwahl, indem Code-SwitchingPhänomene vor allem auf ihre emblematische Funktion hin untersucht werden.

Im Folgenden soll der Zusammenhang von Spracheinstellungen, Ortseinstellungen, Biografie und darauf bezogenen Identitätskonstruktionen am stereotyp ausgeprägten Themenkomplex „Niederdeutsch als Hafensprache“/,Niederdeutsch spricht man im Hafen“ erläutert werden. In einem ersten Schritt werden verschiedene Ausprägungen dieses Wissensbestandes präsentiert, um dann in einem zweiten Schritt erfahrungsbasierte individuelle Konzeptualisierungen exemplarisch zu beschreiben. Drittens ist der identitätsstiftende Zusammenhang von Spracheinstellungen, Ortseinstellungen und biografischen Erfahrungen zu erörtern.

12 Sprachgebrauch, Sprachbewertungen, Spracherwerb, Sprachwandel, Sprachpflege, Ortswissen, Ortsbewertungen.

13 Kindheit, Schulzeit, Ausbildung, Berufsleben, Ruhestand. 


\subsection{Stereotypes Konzept: „Also im Hafen wird schon noch viel Plattdeutsch gesprochen“"}

In der großen Mehrheit der Fälle wird die Zuschreibung „Niederdeutsch spricht man im Hafen“ als Weltwissen formuliert, selten aber als pauschale und uneingeschränkte Aussage, wie von GP 51 (w, *1941), die als spontane Assoziation mit dem Plattdeutschen u.a. den Hafen nennt:

GP 51: Auch noch am Hafen. I: Hmhm. GP 51: Und wenn du da nich Plattdeutsch sprichst, dann kannst das alles gleich vergessen. (97-100)

In der Regel wird die generelle Gültigkeit der Aussage von den Interviewpartnern partiell eingeschränkt, indem ein temporaler Bezug auf die Vergangenheit hergestellt wird. Auf die Frage, wo man heute noch in Hamburg Plattdeutsch hört, nennen GP $11\left(\mathrm{~m},{ }^{\star} 1930\right)$ und GP $12\left(\mathrm{~m},{ }^{\star}\right.$ 1935) den Hafen generell und insbesondere die Werften:

GP 12: $\quad$ Also im Hafen wird schon noch viel Plattdeutsch gesprochen. GP 11: Im Hafen noch viel. GP 12: Auf den Werften und so, GP 11: Ja. GP 12: das is schon noch... (335-339).

Auch wenn die Aussage durch die Partikel „schon“ bekräftigt wird, ist durch das Adverb „noch“ angedeutet, dass der Gebrauch des Niederdeutschen rückläufig ist. Ganz ähnlich erfolgt eine temporale Einordnung bei anderen Gewährspersonen durch negative Vergleiche mit der Vergangenheit („nicht mehr“ [GP 68], „nich mehr so viel“ [GP 10], „wirklich sehr viel weniger“ [GP 10], „bis vor einiger Zeit noch“ [GP 66]).

Einen unsicheren epistemischen Status der Aussage „Niederdeutsch spricht man im Hafen“ belegen häufige Subjektivierungen durch „,ich meine“ (GP 04), in anderen Interviews „ich kann mir vorstellen“ (GP 39), „denke ich“ (GP 71) oder durch Adverbien „wahrscheinlich“ (GP 04), „vielleicht“ (GP 71, GP 73) und „sicherlich“ (GP 35).

GP 04: Nich, aber ich meine, [GP 03: Ja.] die/ die/ die äh äh klassische [GP 03: Hafenarbeiter.] Hafenarbeitersprache is es wahrscheinlich nicht mehr, weil es den klassischen Hafenarbeiter gar nich [I: Ja.] mehr gibt. [...] Das sind doch alles Spezialisten, die [GP 03: Ja.] da auf ihrm... [...] Also das sind ja eher Computerspezialisten als/ als äh Säckeschlepper. [GP 03: (Die fahren da äh ohne Anhang.)] (342-362) 
Begründet wird die Einschränkung des Gebrauchs durch die neuere Entwicklung auf dem Arbeitsmarkt, z.B. durch die zunehmende Digitalisierung. „Klassischen“ Hafenarbeitern, die traditionelle Berufe im Hafen ausüben, wie den Schauerleuten, hier als „Säckeschlepper“ bezeichnet, werden von GP 04 (m, ^1938) „Computerspezialisten“ gegenübergestellt. Dabei wird das Niederdeutsche der traditionellen Arbeitssphäre zugeordnet, das im Laufe des Wandels der Hafenberufe immer weniger verwendet wird. Andere Gewährspersonen (GP 71, w, *1956) machen die Internationalisierung des Arbeitsmarktes für den Sprachwandel verantwortlich oder schränken die Sprechergruppe auf die „Ureinwohner“ bzw. „AltHamburger“ ein (GP 35, m, *1950).

Auffällig sind die Gegenüberstellungen von Sprechergruppen traditioneller und neuer Berufe, von alt und jung, alteingesessen und international. Bei der Formulierung von Erfahrungswissen in anderen Passagen kommt eine Einschränkung des kommunikativen Geltungsbereichs hinzu. Niederdeutsch wird aktuell nicht mehr als Sprache im Beruf oder in der Öffentlichkeit wahrgenommen.

\subsection{Erfahrungsbericht: „Im Freihafen hat man sich nur plattdeutsch unterhalten“}

Von den Gewährspersonen, die selbst im Hafen gearbeitet haben, wird die Zuschreibung des Niederdeutschen als Hafensprache als Erfahrungswissen geschildert, das generalisiert wird.

GP 58: Ja, dann äh war das eben so in den fünfziger Jahren, äh ich hab dann eine Lehre gemacht, Im- und Export, im Freihafen. I: Hmhḿ. GP 58: Im Freihafen musste man Plattdeutsch sprechen. Wenn man im Freihafen kein Plattdeutsch sprechen konnte, dann stand man immer hinten dran. I: Hmhḿ. GP 58: Das heißt, man war immer der Letzte, der dran kam. Die Quiddjes, die kamen immer zum/zum Schluss. Das heißt also, da kam eim die plattdeutsche Sprache... War ein Riesenvorteil, wenn man also Plattdeutsch konnte [...]. (36-40)

GP 58 (m, *1941) berichtet von den Vorteilen, die er als kompetenter Niederdeutsch-Sprecher während seiner Lehrzeit in den 1950er-Jahren hatte. Er beginnt seine Erzählung mit der entsprechenden zeitlichen, räumlichen und situativen Verortung. Niederdeutsch-Kenntnisse bezeichnet er in der damaligen Zeit im Hamburger Hafen zusammenfassend als unabdingbar, was durch das Modalverb „müssen“ sowie durch das Konditionalgefüge hervorgehoben wird, in dem die 
negativen Konsequenzen einer fehlenden Sprachkompetenz genannt werden. Generalisiert wird die Aussage durch das Indefinitpronomen „man“. Kontrastierend werden die beruflich benachteiligten Nicht-Niederdeutsch-Kompetenten als „Quiddjes“ bezeichnet. ${ }^{14}$ An einer anderen Stelle bezeichnet GP 58 Niederdeutsch explizit als Hafensprache:

GP 58: Da wurde [I: Okay.] über alles nur in Plattdeutsch unterhalten. [I: Hmhḿ.] Im Freihafen hat man sich nur Plattdeutsch unterhalten. [I: Hmhḿ.] ((.)) Die Gespräche am Tisch, beim Frühstück, beim Mittach waren in Plattdeutsch. [...] Äh im Hafen ging das, I: Ja. GP 58: weil es is ((.)) Plattdeutsch is ne Hafensprache. ((lacht kurz)) [I: Hmhḿ. Hm.] Also da war es überhaupt gar nicht schwierig, weil/ [I: Okay.] äh weil die Begriffe äh im Hamburger Hafen, das war ja kommt/ kommt/ das kommt ja aus der plattdeutschen Sprache. [I: Hmhḿ.] Also insofern gabs da gar keine ((.)) Schwierigkeiten. [I: Hm.] ((holt Luft)) Nä [I: Okay. ((holt Luft))] $n$ Hoken oder Gripen, oder wat das anners is, dat is Plattdüütsch. I: Ja. Das gabs alles dann. GP 58: Na das gabs alles so. (252-281)

Die generelle Gültigkeit der Aussage wird sowohl durch das Adverb „nur“, aber auch durch beispielhaft angeführte Gesprächssituationen dargestellt. Nach der expliziten Zuschreibung („Plattdeutsch is ne Hafensprache“) lacht die GP kurz auf und signalisiert damit den epistemischen Status der Aussage. Die Tauglichkeit des Niederdeutschen als Arbeitssprache im Hafen begründet er dennoch für die Interviewerin im folgenden Kausalsatz, indem er auf die niederdeutsche Herkunft der Terminologie aufmerksam macht („das kommt ja aus der plattdeutschen Sprache“). Hier setzt er sein metasprachliches Wissen ein, belegt die Aussage mit niederdeutschen Ausdrücken aus dem Berufsjargon (Hoken, Griepen $=$ Sackhaken) und bestätigt auch auf Niederdeutsch noch einmal die sprachliche Einordnung („dat is Plattdüütsch“). GP 58 zeigt sich hier durch den Inhalt der Aussage und durch das Code-Switching als sprachlich versiert - auch gegenüber der Interviewerin. Er kann ihr als Sprachwissenschaftlerin sein laienlinguistisches Wissen mitteilen und positioniert sich damit ihr gegenüber, nicht zuletzt auch durch sein Lachen, als Sprachexperte, der in diesem Moment überlegen ist.

14 Nach dem Hamburgischen Wörterbuch steht „Quiddje“ für „,jem., der nicht (richtig) Platt spricht, Fremder, in Hbg. Zugereister, Nichthamburger“ (Hamburgisches Wörterbuch 1956-2006, Bd. 3, Sp. 929). 


\subsection{Einstellung, Biografie und Identitätskonstruktion: Hamburg - Hafen - Plattdeutsch}

Die Stadt Hamburg, der Hafen und das Hafen(arbeiter)milieu werden von GP 58 explizit als Teil der eigenen Identität beschrieben. Er bringt seine enge emotionale Verbindung zur Stadt zum Ausdruck, wenn er, seine soziale Identität konstruierend, schildert, dass ihn die Erfahrungen in seiner Jugend ,geprägt“ haben (,ich mag Hamburg, also das ist schon irgendwo es gehört auch zu mir irgendwo. [...] Hat mich auch geprägt“ [947-951]). Auf die Frage, welche spontanen Assoziationen mit Hamburg verbunden werden, antwortet er:

GP 58: ((holt tief Luft)) Ja, türlich gibt das son paar Sachen, äh eh, Teile/ an und für sich, aber auch Teile, die es heute einfach nicht mehr gibt äh. ((.)) Für mich war ((.)) allein der Freihafen, das is für mich Hambuich gewesen. I: Hmhḿ. GP 58: Die Speicherstadt [klopft auf den Tisch] is für mich Hambuich [klopft auf den Tisch] gewesen, wo das also im Grunde genommen, wo das Leben tobte, wo man äh das ausländische Gewürz roch, wo man I: Hmhḿ. GP 58: also äh die, die große Welt spürte, ((.)) das is für mich I: Ja. GP 58: Hambuich gewesen. Und is heute noch für mich Hambuich, nur das hat sich ja nun auch schon verändert. (989-995)

Hier fungiert der Hamburger Hafen als ein wesentliches Element für die eigene lokale Identitätsbildung. Die Bezugnahme auf die Vergangenheit steht im Vordergrund, wie es explizit in dreifacher Wiederholung der Verbalphrase mit entsprechender Tempuswahl deutlich wird (,is ... gewesen“). Der Sprecher schildert seine individuelle Wahrnehmung der Stadt (,für mich“) durch den Bezug auf Freihafen und Speicherstadt, die Hafengebiete, in denen GP 58 in seiner Lehrzeit selbst Niederdeutsch gesprochen hat. Hamburg, Hafen und Niederdeutsch sind für GP 58 eng miteinander und biografisch mit seiner eigenen Person verknüpft. Auch wenn der traditionelle Hafen nicht mehr existiert, bleibt der positive emotionale Bezug bestehen, da GP 58 sich selbst als „Wassermensch“ bezeichnet, für den der Hafen ein „Bezugspunkt“ ist (,Und das is ((.)) für mich auch irgendwo ein Stück, wo ich ein/ auch Bezugspunkt, ich bin Wassermensch, also ich bin einer, der wahnsinnig gern Wasser irgendwo in der Nähe hat“ [1003]).

Auch in der bereits eingangs zitierten Passage wird die Verbindung von Sprache, Stadt, Hafen und Hafenmilieu explizit thematisiert. GP 58 bedauert in diesem Zusammenhang, dass die Sprache möglicherweise verschwinden könnte: 
GP 58: Ja, ich sag einfach mal, Plattdeutsch hat für Hambuich schon eine Bedeutung ((.)) ähm Plattdeutsch ist auch ein Stück Hambuich. Und meines Erachtens gehört die plattdeutsche Sprache ähm die gehört einfach zu Hambuich, [I: Hmhḿ.] die gehört einfach auch zum Hafen, die gehört einfach zum Milieu I: Ja. GP 58: Hambuich [I: Hmhḿ.] gehört diese Sprache einfach dazu. Und es wär also wirklich jammerschade, [klopft auf den Tisch] wenn dieses Milieu und dieses äh dieses Stück Hambuich, das al/ an und für sich das Herz ist, I: Hmhḿ. GP 58: wenn das verloren gehen würde [klopft mit einem Gegenstand auf den Tisch]. I: Hm. GP 58: Das ist so meines Erachtens, wäre das wirklich jammerschade. Es geht ((.)) ein Stück Kulturgut verloren. (896-904)

Niederdeutsch wird als ein spezifisches Hamburger Kulturgut aufgefasst, als Ingredienz des Hafens und des Hafenmilieus. Die Bedeutung dieses Zusammenhangs wird dadurch betont, dass das „Milieu“, dessen Teil das Niederdeutsche darstellt, als „Herz“ der Stadt metaphorisiert wird. Die eigene emotionale Verbundenheit wird im nachdrücklichen Bedauern über ein mögliches Verschwinden der Sprache verbalisiert (,wirklich jammerschade“). Die niederdeutsche Sprache wird - auch evoziert durch den Vergleich mit einer verbrannten Bibliothek - als bedrohtes Kulturgut konzeptualisiert („Da geht viel Kultur verloren. Das ist so, als wenn man äh ne ganze Bibliothek ((.)) verbrennt.“ [908]).

\section{Niederdeutsch-Konzepte}

Die Analysen zeigen die Virulenz von Zuschreibungen und die Konstanz von Einstellungsmustern. Niederdeutsch ist Teil eines traditionellen Hamburg-Konzepts, das bis heute als Stereotyp gültig geblieben ist. Die Sprache wird von vielen Interviewten generell als Sprache des Hamburger Hafens konzeptualisiert und dabei positiv bewertet. Bei solchen Zuschreibungen bleibt jedoch der epistemische Status unsicher, und dem stereotypen Weltwissen „Niederdeutsch spricht man im Hafen“ wird das eigene Erfahrungswissen gegenübergestellt, nämlich dass mit dem tiefgreifenden Wandel der Arbeitswelt im Hafen ein sprachlicher Wandel einhergegangen ist und Niederdeutsch in der Gegenwart im Hafen und in hafennahen Stadtteilen nicht mehr in der Öffentlichkeit zu hören ist. Zudem wird in den Berichten auch der Funktionswandel des Niederdeutschen von einer Berufssprache zu einer Nahsprache deutlich. Dennoch bleiben die generalisierten Zuschreibungen erhalten, die biografisch verankert und emotional aufgeladen sind. Das vergangene Erleben, das zu einer erzählenswerten Anekdote geronnen ist, wirkt 
sich nach wie vor auf die aktuellen Sprachkonzepte aus. Verschiedene semantisch-pragmatische Operationen (Generalisierungen, Subjektivierungen, Kontrastierungen) prägen die Erzählungen, die zur Begründung und Illustration einer Zuschreibung dargeboten werden, und verdeutlichen die einzelnen Facetten der Einstellungen sowie das Selbstbild, woran auch Positionierungen beteiligt sind. Durch Code-Switching ins Niederdeutsche wird die erzählte Situation verlebendigt, aber auch die eigene Kompetenz des Sprechers unter Beweis gestellt.

\section{Literatur}

Adler, Astrid et al. (2016): Status und Gebrauch des Niederdeutschen 2016. Erste Ergebnisse einer repräsentativen Erhebung. Mannheim.

Allport, Gordon (1935): Attitudes. In: Murchison, Carl (Hg.): A Handbook of Social Psychology. Worcester, MA, S. 798-844.

Berger, Peter/Luckmann, Thomas (2010): Die gesellschaftliche Konstruktion der Wirklichkeit. Eine Theorie der Wissenssoziologie. 23. Aufl. Frankfurt a.M. [1. Aufl. Frankfurt a.M. 1966].

Betten, Anne (2017): Plädoyer für Themen- und Textsortenvariation in sprachbiographischen Interviews. Am Beispiel von Aufnahmen mit deutschsprachigen Emigranten in Israel. In: Schröder, Ingrid/Jürgens, Carolin (Hg.): Sprachliche Variation in autobiographischen Interviews. Theoretische und methodische Zugänge. Frankfurt a.M. u.a., S. 167-197.

Bieberstedt, Andreas (2017): Lebenslauf und Sprachbiographie. Versuch einer sprachbiographischen Modellbildung aus dialektologischer Perspektive. In: Schröder, Ingrid/Jürgens, Carolin (Hg.): Sprachliche Variation in autobiographischen Interviews. Theoretische und methodische Zugänge. Frankfurt a.M. u.a., S. 47-80.

Blotevogel, Hans Heinrich (2001): Regionalbewusstsein und Landesidentität am Beispiel von Nordrhein-Westfalen. In: Institut für Geographie Universität Duisburg. Diskussionspapier 2. Duisburg, S.1-17. Internet: http://duepublico.uni-duisburg-essen.de/servlets/ DerivateServlet/Derivate-5198/blotevogel2.pdf (Stand: 27.4.2018).

Cuonz, Christina (2014): Was kann die diskursive Spracheinstellungsforschung (nicht)? Methodologische und epistemologische Überlegungen. In: Cuonz, Christina/Studler, Rebekka (Hg.): Sprechen über Sprache. Perspektiven und neue Methoden der Spracheinstellungsforschung. Tübingen, S. 31-64.

Eagly, Alice/Chaiken, Shelly (1998): Attitude structure and function. In: Gilbert, Daniel/Fiske, Susan/Lindzey, Gardner (Hg.): The Handbook of Social Psychology. Bd. 1. 4. Aufl. New York, S. 269-322.

Eckert, Penelope (2012): Three Waves of Variation Study. The Emergence of Meaning in the Study of Sociolinguistic Variation. In: Annual Review of Anthropology 41, S. 87-100.

Ehlich, Konrad (1983): Alltägliches Erzählen. In: Sanders, Willy/Wegenast, Klaus (Hg.): Erzählen für Kinder - Erzählen von Gott. Begegnungen zwischen Sprachwissenschaft und Theologie. Stuttgart u.a., S. 128-150.

Eichinger, Ludwig M. (1999): Mehrsprachigkeit im Kontext. Kontexte individualisierter Sprachenwahl. In: Zeitschrift für Angewandte Linguistik 30, S. 41-54. 
EXMARaLDA. Internet: www.exmaralda.org (Stand: 1.5.2018).

Fischer, Lorenz/Wiswede, Günter (2009): Grundlagen der Sozialpsychologie. 3., neu bearb. Aufl. München.

Flender, Armin/Pfau, Dieter/Schmidt, Sebastian (2001): Regionale Identität zwischen Konstruktion und Wirklichkeit. Eine historisch-empirische Untersuchung am Beispiel des Siegerlandes. Baden-Baden.

Garrett, Peter (2010): Attitudes to Language. Cambridge.

Gergen, Kenneth/Gergen, Mary (1988): Narrative and the Self as Relationship. In: Berkowitz, Leonard (Hg.): Advances in Experimental Social Psychology. New York, S. 17-56.

Hamburgisches Wörterbuch (1956-2006). Auf Grund der Vorarb. von Christoph Walther und Agathe Lasch hrsg. von Beate Hennig und Jürgen Meier. 5 Bde. Neumünster.

Hess-Lüttich, Ernest (2004): Die sozialsymbolische Funktion der Sprache. In: Ammon, Ulrich et al. (Hg.): Soziolinguistik. Ein internationales Handbuch zur Wissenschaft von Sprache und Gesellschaft. 1. Teilbd. 2., vollst. neu bearb. u. erw. Aufl. Berlin/New York, S. 491-502.

Hügli, Anton (2010): Identität. In: Bermes, Christian/Dierse, Ulrich (Hg.): Schlüsselbegriffe der Philosophie des 20. Jahrhunderts. (= Archiv für Begriffsgeschichte, Sonderheft 6). Hamburg, S. 131-148.

Jürgens, Carolin (2015): Niederdeutsch im Wandel. Sprachgebrauchswandel und Sprachwahrnehmung in Hamburg. Hildesheim.

Jürgens, Carolin (2016): Regionale Identität per Einkaufstüte. Eine Fallstudie zum Enregisterment des Niederdeutschen in Hamburg. In: Bieberstedt, Andreas/Ruge, Jürgen/ Schröder, Ingrid (Hg.): Hamburgisch. Struktur, Gebrauch und Wahrnehmung der Regionalsprache im urbanen Raum. Frankfurt a.M., S. 307-343.

Kaiser, Hansruedi (2010): Arten des Wissens. Internet: www.hrkll.ch/typo/fileadmin/Texte/ ILM/arten_des_wissens.pdf (Stand: 27.4.2018).

König, Katharina (2014): Spracheinstellungen und Identitätskonstruktion. Eine gesprächsanalytische Untersuchung sprachbiographischer Interviews mit Deutsch-Vietnamesen. Berlin.

König, Katharina (2017): Das sprachbiographische Interview als Interaktion - Eine gesprächsanalytische Perspektive auf ein Forschungsinstrument. In: Schröder, Ingrid/Jürgens, Carolin (Hg.): Sprachliche Variation in autobiographischen Interviews. Theoretische und methodische Zugänge. Frankfurt a.M. u.a., S. 199-223.

Krappmann, Lothar (2004): Identität. In: Ammon, Ulrich et al. (Hg.): Soziolinguistik. Ein internationales Handbuch zur Wissenschaft von Sprache und Gesellschaft. 1. Teilbd. 2., vollst. neu bearb. u. erw. Aufl. Berlin/New York, S. 405-412.

Kraus, Wolfgang (2002): Falsche Freunde. In: Straub, Jürgen/Renn, Joachim (Hg.): Transitorische Identität. Der Prozesscharakter des modernen Selbst. Frankfurt a.M., S. 159-186.

Kresić, Marijana (2006): Sprache, Sprechen, Identität. Studien zur sprachlich-medialen Konstruktion des Selbst. München.

Lasagabaster, David (2004): Attitude. In: Ammon, Ulrich et al. (Hg.): Soziolinguistik. Ein internationales Handbuch zur Wissenschaft von Sprache und Gesellschaft. 1. Teilbd. 2., vollst. neu bearb. u. erw. Aufl. Berlin/New York, S. 399-405.

Lucius-Hoene, Gabriele/Deppermann, Arnulf (2002): Rekonstruktion narrativer Identität. Ein Arbeitsbuch zur Analyse narrativer Interviews. Opladen.

Lucius-Hoene, Gabriele/Deppermann, Arnulf (2004): Narrative Identität und Positionierung. In: Gesprächsforschung - Online-Zeitschrift zur verbalen Interaktion 5, S. 166-183. Internet: www.gespraechsforschung-ozs.de/heft2004/ga-lucius.pdf (Stand: 27.4.2018). 
Mühler, Kurt/Opp, Karl-Dieter (2008): Ursachen für die Identifikation von Bürgern mit ihrer Region und Wirkungen auf ihr individuelles Handeln. Abschlussbericht. Leipzig. Internet: www.kulturregionen.org/2008_symposium/muehler-opp_zusammenfassung_regionale_ Identifikation.pdf (Stand: 27.4.2018).

Neuland, Eva (1993): Sprachgefühl, Spracheinstellungen, Sprachbewusstsein. Zur Relevanz „subjektiver Faktoren“ für Sprachvariation und Sprachwandel. In: Mattheier, Klaus et al. (Hg.): Vielfalt des Deutschen. Festschrift für Werner Besch. Frankfurt a.M. u.a., S. 723747.

Neumann, Lara/Schröder, Ingrid (2017): Identitätskonstruktionen in sprachbiographischen Interviews. Analysen zur Funktion des Niederdeutschen in Hamburg. In: Schröder, Ingrid/ Jürgens, Carolin (Hg.): Sprachliche Variation in autobiographischen Interviews. Theoretische und methodische Zugänge. Frankfurt a.M. u.a., S. 225-242.

Quasthoff, Uta (1987): Linguistic Prejudice/Stereotypes. In: Ammon, Ulrich et al. (Hg.): Soziolinguistik. Ein internationales Handbuch zur Wissenschaft von Sprache und Gesellschaft. 1. Halbbd. Berlin/New York, S. 785-799.

Riehl, Claudia (2000): Spracheinstellungen und Stereotype im Lichte diskursiver Praxis. In: Deminger, Szilvia et al. (Hg.): Einstellungsforschung in der Linguistik und Nachbardisziplinen. Frankfurt a.M. u.a., S. 141-160.

Rosa, Hartmut (2007): Identität. In: Straub, Jürgen/Weidemann, Arne/Weidemann, Doris (Hg.): Handbuch interkulturelle Kommunikation und Kompetenz. Grundbegriffe - Theorien Anwendungsfelder. Stuttgart, S. 47-56.

Schröder, Ingrid (2010): Plattdeutsch in Hamburg. Sprachwahl als Mittel zur Konstruktion lokaler Identität? In: Müns, Wolfgang (Hg.): Man mag sik kehrn un kanten, as man will, noch jümmer is der’n Eck, wo man ni wen is. 100. Jahrgang der Zeitschrift „Quickborn“. Festschrift. Hamburg, S. 585-601.

Schröder, Ingrid (2013): Sprache, Stadt und Stereotyp. Zur sozialsymbolischen Funktion des Niederdeutschen im urbanen Raum. In: Żebrowska, Ewa/Glaser, Elvira (Hg.): Deutsche Dialekte und Regionalsprachen. Frankfurt a.M., S. 377-382.

Schröder, Ingrid/Jürgens, Carolin (2016): Sprachstereotype und ihre Realisierungen im Gespräch. In: Bieberstedt, Andreas/Ruge, Jürgen/Schröder, Ingrid (Hg.): Hamburgisch. Struktur, Gebrauch, Wahrnehmung der Regionalsprache im urbanen Raum. Frankfurt a.M. u.a., S. 345-385.

Schröder, Ingrid/Jürgens, Carolin (2017): Einstellungen gegenüber regionalen Sprachformen in der Großstadt: Niederdeutsch in Hamburg (NiH). Eine Projektskizze. In: Dies. (Hg.): Sprachliche Variation in autobiographischen Interviews. Theoretische und methodische Zugänge. Frankfurt a.M. u.a., S. 11-46.

Silverstein, Michael (2003): Indexical order and the dialectics of sociolinguistic life. In: Language \& Communication 23, S. 193-229.

Straub, Jürgen (2000): Identitätstheorie, Identitätsforschung und die postmoderne armchair psychology. In: Zeitschrift für Qualitative Bildungs-, Beratungs- und Sozialforschung 1, 1, S. 167-194.

Straub, Jürgen (2011): Identität. In: Jaeger, Friedrich/Liebsch, Burkhard (Hg.): Handbuch der Kulturwissenschaften. Bd. 1: Grundlagen und Schlüsselbegriffe. Stuttgart u.a., S. 277303.

Tajfel, Henri (1981): Human Groups and Social Categories. Studies in Social Psychology. Cambridge. 
Thim-Mabrey, Christiane (2003): Sprachidentität - Identität durch Sprache. Ein Problemaufriss aus sprachwissenschaftlicher Sicht. In: Janich, Nina/Thim-Mabrey, Christiane (Hg.): Sprachidentität. Identität durch Sprache. (= Tübinger Beiträge zur Linguistik 465). Tübingen, S. 1-18.

Tophinke, Doris (2002): Lebensgeschichte und Sprache. Zum Konzept der Sprachbiographie aus linguistischer Sicht. In: Bulletin suisse de linguistique appliquée 76, S. 1-14.

Tophinke, Doris/Ziegler, Evelyn (2006): „Aber bitte im Kontext!“ Neue Perspektiven der dialektologischen Einstellungsforschung. In: Voeste, Anja/Gessinger, Joachim (Hg.): Dialekt im Wandel. Perspektiven einer neuen Dialektologie. (= Osnabrücker Beiträge zur Sprachtheorie 71). Duisburg u.a., S. 205-224.

Wirrer, Jan (2017): Sprecherbiographie, soziales Alter und kommunikative Netzwerke. In: Schröder, Ingrid/Jürgens, Carolin (Hg.): Sprachliche Variation in autobiographischen Interviews. Theoretische und methodische Zugänge. (= Sprache in der Gesellschaft 35). Frankfurt a.M. u.a., S. 81-104. 